\title{
THE COOLING OF OPTICAL GLASS MELTS.
}

By Howard S. Roberts.

When a melt of glass is removed from the melting furnace, it may be either left to cool in the pot, or be cast and rolled into a plate. This paper has to do with the former case, where the melt is cooled in the pot.

The choice between these two methods is a question of the quality of glass desired and of expediency. When the melt is set out of the furnace it is never entirely homogeneous. The middle is usually free from contamination, but near the bottom and sides of the pot, the glass is badly contaminated with material dissolved from the pot. If the melt is cooled in the pot, this contaminated glass need not be much disturbed, but where it is cast and rolled into a sheet, it is apt to be spread rather evenly through the whole plate.

While the process of cooling in the pot involves a greater amount of labor and waste in the mechanical preparation of the glass, it can be made to yield glass of the highest quality. The rolling process, on the other hand, may give a large yield of glass that is entirely suitable for many, if not most, optical instruments.

Where the melt is cooled in the pot, the conditions to be sought are: (I) that the cooled melt shall break into large, reasonably rectangular blocks, having smooth faces; and (2) that not much glass shall be spoiled by inhomogeneity, $i$. e., by striae, bubbles, crystals or devitrification.

It is not feasible to cool the melt so slowly that it will remain unbroken; nor is this particularly desirable because of the necessity of breaking it up later for examination and for rejection of defective portions.

At the melting temperature, glass is a fairly mobile liquid. As it cools its viscosity increases continuously, until at ordinary temperatures, though probably not infinite, the viscosity is so great that we apparently have an elastic solid. For convenience 
we shall divide the whole range of cooling into three stages: The Stage of Fluidity; The "Annealing Range;" The Final Stage.

\section{Stage of Fluidity.}

During this stage, the glass is apt to be spoiled by striae due to convection; by the formation of vacuum bubbles, and, in certain glasses, by the formation of one or more crystalline phases in the glass itself.

Convection.- On leaving the furnace, the glass is still quite fluid, having been stirred until the time of setting out. The portion close to the sides and bottom of the pot is richer in material dissolved from the pot and has an index of refraction different from that of the glass nearer the center. As the melt cools convection currents tend to draw streamers of this contaminated glass upward through the middle of the melt. Below the "setting-out temperature" the glass is too viscous to readily assimilate these streamers by solution; so that, having once appeared, they remain as "striae" or "ream." Particles of foreign material, such as bits of the pot, may also be carried to the middle of the melt where they usually float upward or settle, dragging tails of contaminated glass behind them. Convection continues at a decreasing rate as viscosity increases on cooling, and soon becomes inconsiderable.

As convection is caused by the settling of denser, cooler glass, it can be reduced by insulating the upper portion of the melt, and cooling rapidly from the bottom. Unfortunately, present melting practice usually insures that the bottom of the pot, on leaving the furnace, shall be at a somewhat higher temperature than any other portion; moreover, the bottom is usually made much thicker than the sides. Thus it has not been found possible entirely to prevent convection even by immersing the bottom of the pot in a pan of water.

It should be emphasized that measures to reduce convection should be taken immediately on setting the pot out of the furnace. The surface of the melt should immediately be covered with a layer of kieselguhr or other light insulator; and the pot be placed on bricks as quickly and with as little agitation of the glass as possible. 
Vacuum Bubbles.-Another phenomenon peculiar to this stage is the formation of vacuum bubbles. These are caused by the premature "setting" of the top surface of the melt, thus enclosing the inner portion in a rigid envelope. On further cooling, the contraction of the interior greatly enlarges any small bubbles that may be present and may even draw in considerable quantities of air through cracks in the pot. This effect can be entirely prevented by insulating the top surface of the glass sufficiently to prevent the temperature of that portion from falling much below the temperature of the center of the melt.

Crystallization.- While the glass is yet soft, conditions are particularly favorable for the formation of crystalline phases in the melt, giving rise to the phenomenon of "crystallization". or "devitrification."1 In addition, undissolved particles of sand or of the pot may form nuclei for the deposition of crystalline material from the glass. Given time enough at a suitable temperature, one or more crystalline phases may separate from any glass, but this effect begins reluctantly and proceeds slowly, so that most glasses can be cooled rapidly enough to prevent it.

Conditions to be Secured during Stage of Fluidity.-To inhibit convection and the formation of bubbles, the surface of the glass should be kept warm and heat removed chiefly from the bottom. The cooling should be made rapid down to a temperature below that at which there is no further danger of crystallization or devitrification. Convection can be further reduced by intensive chilling of the bottom of the pot by means of a current of compressed air or a fine stream of water, during the last hour before it is removed from the melting furnace.

\section{Annealing Range.}

The rate of cooling through the second stage determines the size and shape of the pieces into which the melt will break. The whole of the cooled melt, except the portion close to the pot, should be found broken into large, reasonably rectangular blocks, having smooth, flat surfaces through which their interior may be examined. Under these conditions the blocks have been

1 N. L. Bowen, J. Am. Ceram. Soc., I, 599 (1918). 
found to break clearly, but where the blocks have curved surfaces, they tend to form wedge-shaped fragments having very rough surfaces when broken. In extreme cases the interior portion of the melt may be found in the form of a large, flattened sphere of glass called a "marble" or "onion," which can seldom be broken satisfactorily.

When cold, the melt is usually found to be cut by cracks of two types: curved cracks, roughly spherical and concentric with the center of the melt, which form shells an inch or two thick in the outer portion of the melt; and plane cracks which divide the melt into large blocks.

Spherical Cracks.-If, as must always be the case, different parts of the melt cool at different rates, the resulting differences in rate of contraction cause stresses in the melt, which in turn tend to make the glass flow in such a way as to relieve the strains set up by them. The velocity of this release of strain decreases as the temperature falls, because of the increase of viscosity on cooling, and is, at any instant, nearly proportional to the total amount of strain causing it. ${ }^{1}$ Consequently the amount of strain present will tend to approach such a value that it will be relieved, by internal flow, at the same rate as that at which fresh strain is introduced. As this flow is not very rapid, even at high temperatures, and is imperceptible at low temperatures, strain persists long after fresh strain ceases to be introduced.

Rather than consider the differences between cooling rates, it is somewhat simpler to speak of the time rate of change of the temperature drop (since the difference between the rates of change of two variables is equal to the rate of change of their difference, this amounts to the same thing). Then, if we assume that the coefficient of linear expansion of the glass is constant over the maximum temperature range throughout the melt at any instant, the differential rate of contraction, that is the time rate at which strain is introduced, is everywhere proportional to the time rate of change of the temperature gradient.

When the melt is set out of the melting furnace, there is little temperature difference through it. On account of convection 1 This question is fully discussed in a paper in preparation by $L$. $H$. Adams and E. D. Williamson. 
and, perhaps, the transfer of considerable quantities of heat through the glass by radiation as well as by conduction, the temperature drop between the center and the outside of the glass does not reach a maximum until the melt has cooled to a low red heat. An idea of this can be secured from Fig. 7, following.

After passing the maximum this temperature drop decreases, and disappears when the melt has cooled to constant temperature.

While the temperature drop is increasing the outer portions of the melt are contracting more rapidly than the inner and the strain consists of radial compression throughout, accompanied by tangential tension in the outer portions and tangential compression in the inner. ${ }^{1}$

After the maximum drop is passed, the inner portions are contracting more rapidly than the outer, and the stresses being introduced consist of radial tension throughout, accompanied by tangential compression in the outer portion and tangential tension in the inner. As the strains now being introduced are the reverse of those introduced before the temperature drop reached its maximum, their first effect is to neutralize the residuum of the strain introduced at that earlier time. On further cooling and reduction of the temperature drop strain builds up again and tends to fracture the glass by curved cracks more or less normal to the direction of the forces of radial tension. ${ }^{2}$ During the earlier part of the annealing range, as has been said, strain is relieved by flow of the glass so much more rapidly than at lower temperatures that it does not accumulate sufficiently to cause cracks. Thus the spherical cracks are entirely due to the later decrease of temperature gradient.

This tendency to fracture increases as we recede from the center, ${ }^{2}$ therefore fractures tend to follow one another, as strain increases, from the outside toward the center of the melt. If no other factor appears, the final form of the melt is a roughly

${ }^{1}$ We here assume that the isothermal surfaces in the melt are spheres. Actually they appear to be roughly spheroidal, approaching the spherical form at the center.

2 The question of the elastic stresses produced by temperature differences in a solid sphere has been discussed by E. D. Williamson, J. Wash. Acad. Sci., 9, 209-217 (1919). 
spherical core, or "marble," of glass surrounded by a series of shells very like those of an onion. The size of this core depends on the course of the temperature gradient.

This effect is shown in Fig. I, which is a photograph of a melt

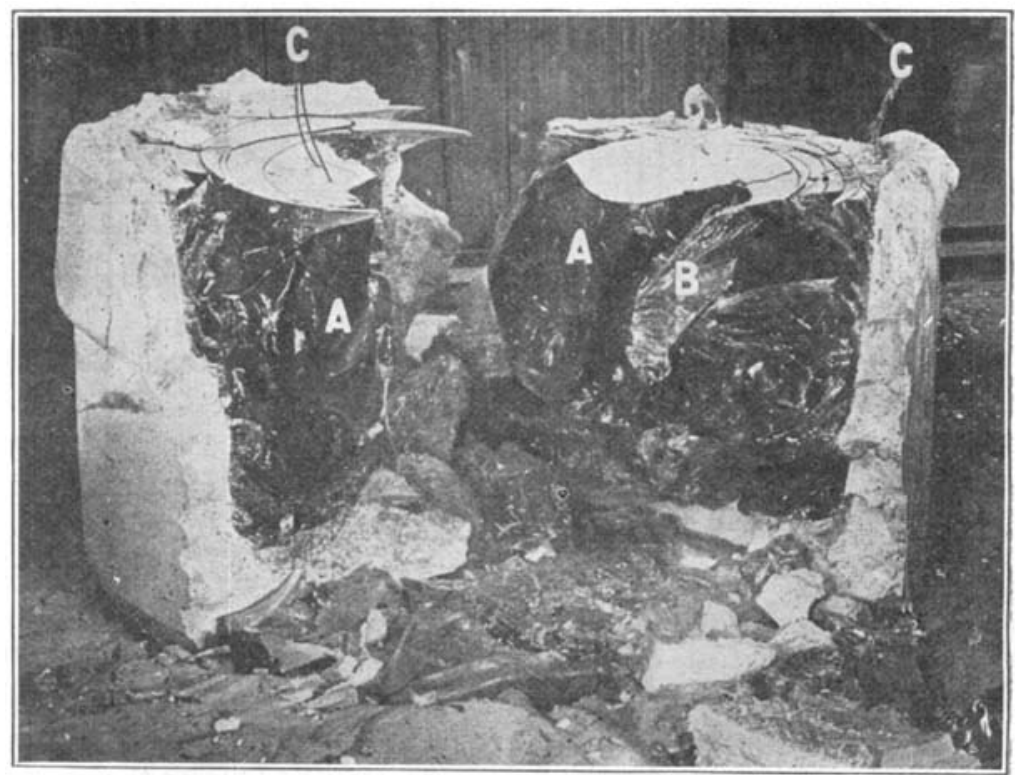

Fig. 1.-Spherical cracks in a poorly annealed melt

that was cooled too rapidly. The cracks here are chiefly of the spherical type, appearing on the top surface as concentric circles. The inner core A (broken in the picture), was quite large, and as is usually the case, includes considerable of the top surface. The shape and irregular surface of the block $B$ are characteristic of poorly annealed melts where the central core is not found unbroken. The wires $\mathrm{C}$ are thermocouples placed in the melt while it was still molten. This is an excellent example of a poorly cooled melt.

Marbles.-Where the central core remains unbroken the resulting marble is often so large as to include not only the top surface of the glass but some of the side walls of the pot as well. 
When it is broken it yields blocks like B in Fig. I; or, in extreme cases, the whole marble may be shattered by a very large number of radial cracks, as in Fig. 2. In this figure the center of the melt was at $C$, and $S$ was the outside surface of the marble.

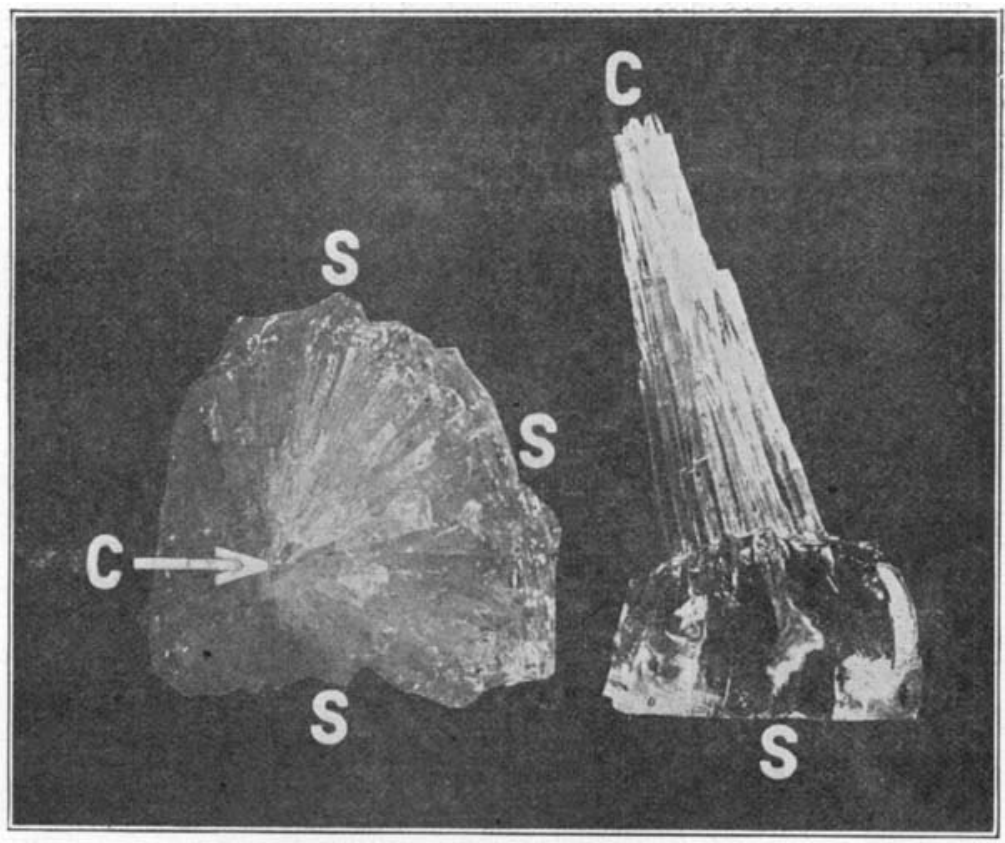

FIG. 2.-Block of glass obtained on breaking a heavily strained marble.

Plane Cracks. - When the melt has cooled to a temperature usually between $200^{\circ}$ and $400^{\circ} \mathrm{C}$, cracks start in the glass at the pot wall. These are probably due to the difference in contraction between the glass and the pot. These cracks extend into the melt until they reach another crack, or the opposite side of the pot. If the interior of the melt has not been separated from the pot by spherical cracks, the first plane crack to appear usually divides the melt in two along a vertical diametral plane. Subsequent cracks are usually not radial, but perpendicular. to the first one. After a comparatively few cracks have formed, 
the tendency for the cracks to follow the shortest path confines their activity to the outer portion of the melt. Here they cut the glass into pieces the size of one's fist or smaller. Horizontal cracks form as well as vertical, but are usually subordinate to the vertical cracks.

The presence of these cracks in the interior not only prevents the formation of a marble by cutting it up before it is formed but also greatly reduces the amount of strain present in the interior.

Fig. 3 is a photograph of a well annealed melt, broken in two

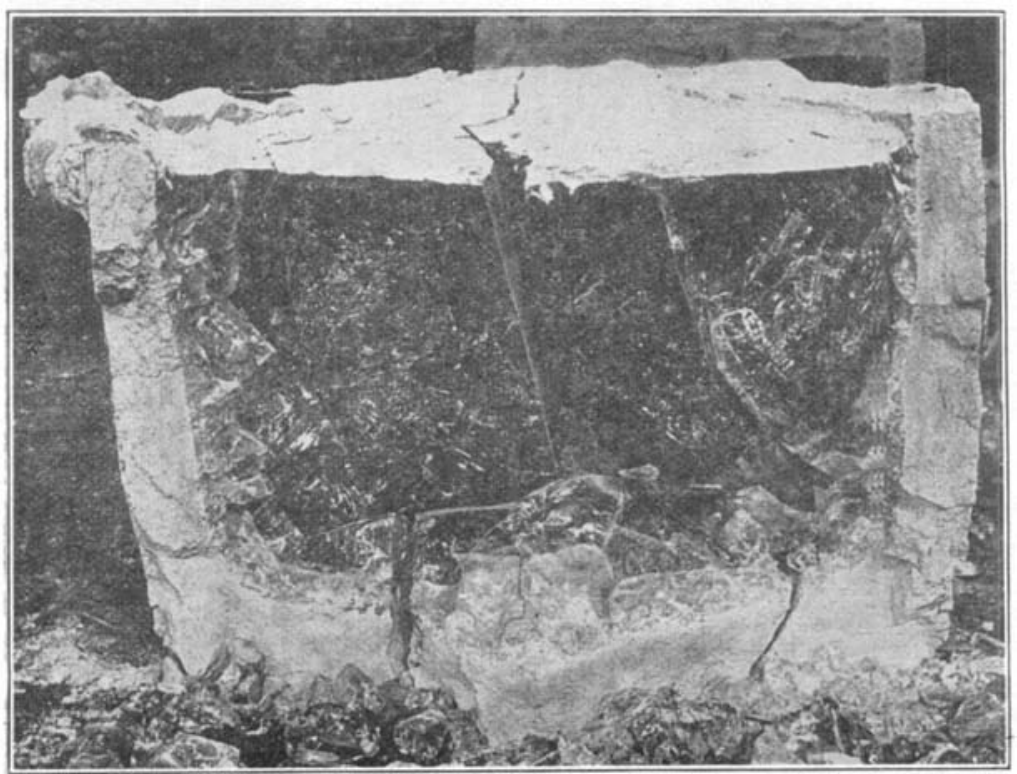

Fig. 3.-Plane cracks in a well-annealed melt.

along the diametral plane crack. The cracks here are entirely of the plane type, the surfaces are smooth and the blocks of glass are large. The smaller fragments are confined to the bottom and sides of the melt where the glass is certain to be full of striae dissolved from the pot.

An experiment was carried out to show the effect of the pres- 
ence of plane cracks on the strain in the melt, and to prove that they were caused by the pot. A "marble" about 20 inches $(50 \mathrm{~cm}$.) in diameter was insulated with a thick layer of sand and heated very slowly to its annealing temperature. It was then cooled to room temperature at a rate somewhat slower than the rate at which 36 -inch $(90 \mathrm{~cm}$.) melts were cooled. Neither spherical nor plane cracks appeared on cooling. On being cut up with a cold chisel, the marble broke into large pieces, but of irregular shape with rather rough surfaces. 36-inch $(90 \mathrm{~cm}$.) pots of glass, although cooled more rapidly than the above, showed plane cracks with smooth surfaces.

The temperature at which the formation of plane cracks begins seems to be nearly independent of the temperature gradient and cooling rate. Therefore it is possible, by controlling the temperature gradient, to hold back the formation of spherical cracks until the plane fracturing is well under way.

Temperature Limits of Annealing Range.-We are now in a position to define the temperature limits of the annealing range, and, from them, those of the other two stages.

If a small block of glass, placed in a polariscope, is heated rapidly, it will exhibit the characteristic colored figure of glass under strain. When the block is passing through a certain temperature range the color changes rather rapidly. We shall call the average temperature of the block at this period the "annealing temperature." 1 It depends on the size of the block and on the rate of heating, but does not vary more than $20^{\circ}$, provided the smallest dimension of the block is less than $\mathrm{I} \mathrm{cm}$. and that it is heated at a rate nearly rapid enough to crack it. The greater part of the strain present in a block of glass held at this temperature will vanish in a few seconds. Thus the extent to which strain can accumulate at this temperature is small compared with the rate at which it is introduced.

This temperature can be more definitely fixed as that at which a given proportion of the strain in a block of a certain size will disappear in a given time. For our purpose a rougher, more easily determined point is sufficient, and preferable.

"The "annealing temperature" determined by this method is considerably higher than the temperature usually employed in the annealing kilns. 
The "annealing temperature" of the glass is here taken as the upper limit of the "annealing range" of the melt (see page 544). The lower limit is taken as the temperature at which the formation of plane cracks begins.

The lower limiting temperature depends on the relation of the coefficient of expansion of the glass to that of the pot. As a first approximation it may be assumed to be $250^{\circ}$ to $300^{\circ}$ below the annealing temperature.

Conditions to be Secured through Annealing Range.-As the spherical cracks are entirely due to the reduction of the temperature gradient, it is best to keep this low in the first place, and then to prevent its decreasing greatly until the formation of plane cracks is well under way. These conditions can be attained by decreasing the cooling rate at a time shortly before the annealing temperature is reached, by adding insulation or otherwise; and by removing the insulation a little while before the end of the annealing range. Removing the insulation will usually increase the temperature gradient temporarily, thereby neutralizing some of the strain present and gaining time for the plane cracks to appear.

\section{Methods of Retarding the Cooling.}

The rate of cooling through the annealing range may be retarded by placing the pot in an inclosed space, such as a kiln or pot-arch, and heating the air around it; or by surrounding the pot with a layer of insulating material. Either method gives a satisfactory result, and there seems to be no great advantage in combining them, except that in any case the surface of the glass should not be exposed, but covered by a layer of insulating material having at least the insulating properties of the pot wall itself.

Either of these methods depends on the fact that the melt tends to cool to the temperature of its surroundings at a rate that is more or less proportional to the difference between its temperature and that of its surroundings. Whether the melt is insulated by the addition of some covering or by the presence of the pot alone, the ratio of cooling rate to temperature difference decreases with increase of insulation. Thus a given rate 
of cooling at any temperature may be attained by adding sufficient insulation at room temperature, or by placing the melt in a sufficiently heated enclosure, with no insulation beside that afforded by the pot and the layer of insulation previously applied to the surface of the glass.

Use of a Heated Kiln or Pot-Arch.-In this method the rate of cooling can be controlled by varying the temperature or the rate at which air is supplied to the pot. Two general methods have been developed: (I) where the temperature and rate are kept nearly constant; and (2), where the temperature and rate are decreased as the melt cools.

In]Fig. 4, A, B, and $\mathrm{C}$ are time-temperature curves for a 36 -inch

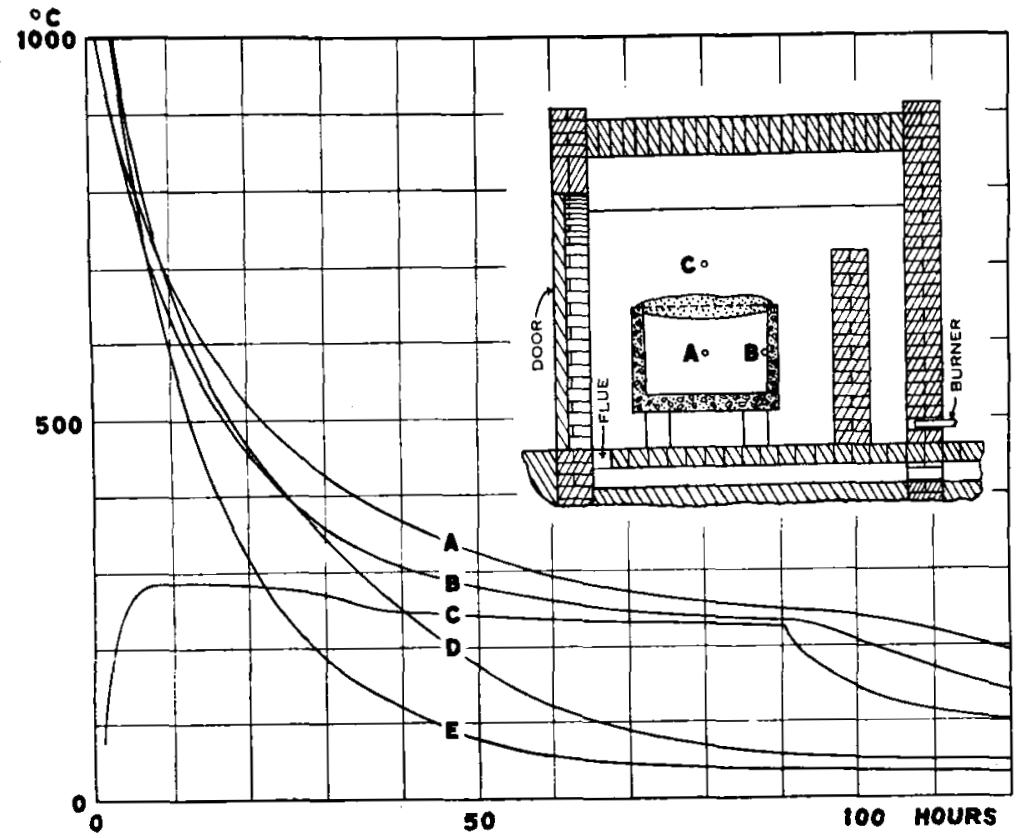

FIG. 4- -Time-temperature curves for melt cooling without added insulation. Curves $A, B$, and $C$ apply to a melt cooled in a heated potarch; Curves $D$ and $E$ to a melt cooled in the open.

(90 cm.) pot of light barium crown glass, $\left(n_{\mathrm{D}}=1.57\right)$ annealed in a heated pot-arch. Curve $A$ represents the temperature at 
the center of the melt; Curve $B$ that at a point on the inside wall of the pot, midway between top and bottom; Curve $\mathrm{C}$ that of the air 8 inches $(20 \mathrm{~cm}$.) above the pot. The top surface of the glass was covered with a 4 -inch (Io $\mathrm{cm}$.) layer of kieselguhr immediately after setting the melt out of the melting furnace. About an hour later the gas was lighted in the arch. Zero time in Fig. 4 and subsequent figures is the time at which the pot was set out of the melting furnace.

Curves $\mathrm{D}$ and $\mathrm{E}$ in the same figure are for a 36 -inch pot of medium flint glass $\left(n_{\mathrm{D}}=\mathrm{I} .6 \mathrm{I}\right)$, insulated on the surface as before, but allowed to cool in the open at a temperature of about $30^{\circ}$. Curve $\mathrm{D}$ represents the temperature at the center of the melt and Curve $\mathrm{E}$ that at a point in contact with the inside of the pot wall midway between top and bottom. In both cases the temperatures were measured by bare-wire thermoelements permanently placed with their junctions at the points indicated.

As the two glasses used in these experiments have not been found to differ widely in their thermal properties, these two sets of curves are comparable and show what effect is to be expected when the temperature to which the melt can cool is raised.

Curves $A$ and $B$ are not only less steep than $D$ and $E$, but closer together, indicating a smaller temperature difference. At the time the gas is shut off in the arch, they begin to diverge again, thus preventing the temperature difference from decreasing below its former minimum until the melt reaches a low temperature.

In the case of Curves $\mathrm{A}, \mathrm{B}$, and $\mathrm{C}$, no attempt was made to cool the melt rapidly through the stage of fluidity. In practice it would be better to let it cool 3 or 4 hours in the open before it is placed in the arch; and to have the arch heated while the pot is waiting rather than after it goes in.

This method was developed with the expectation of building a long, heated tunnel through which the pots should be moved on cars; the length of the tunnel to be such that it should contain three days' run of pots under conditions of maximum production. It was found that if a slow stream of air were supplied to the tunnel at a constant temperature of $300^{\circ} \mathrm{C}$, the annealing would be entirely satisfactory. 
Dr. C. N. Fenner, of the Geophysical Laboratory, developed the following method at the plant of the Spencer Lens Co.: When a melt was removed from the melting furnace, it was allowed to cool freely until a new pot had been taken from an individual pot arch and set into the melting furnace. The melt, having cooled about I 5 minutes in the open and until convection currents had subsided, was then placed in the hot pot-arch from which the new pot had been taken. The burners of the arch were then adjusted so that its temperature would fall to a low red heat $\left(500\right.$ to $\left.600^{\circ}\right)$ over night (about I 6 hours). He then found that the melt would anneal well: (I) if the firing were adjusted so that the temperature of the air would reach about $400^{\circ}$ at the end of another 24 hours, or (2) if the flame were turned completely out. In the latter case, since both the firing ports and flues of the arch were close to the bottom, there was probably no tendency for air to circulate through it, and it cooled solely by the conduction of heat through the walls of the pot-arch. If, however, the flame was turned very low, but not out, a circulation of relatively cool air was induced which caused the melt to cool so rapidly that it was poorly annealed.

No data are available for the temperatures within the melt, but it seems likely that this procedure causes the temperature gradient to remain small, and to reach its maximum at a temperature well within the annealing range. Thus the strains that fracture the melt did not begin to appear until a low temperature was reached.

At the Bausch \& Lomb plant, C. N. Fenner and R. E. Hall developed a method similar to that which was later used at the Spencer Lens plant, except that fire was maintained in the pot arch until it had reached a temperature of $200^{\circ}$ to $300^{\circ}$. This method also probably keeps the temperature gradient low, and increases it again just after the melt has passed out of the annealing range.

These three methods are summarized in the curves of Fig. 5, which represent the temperatures of the air over or close to the pot, plotted against the elapsed time after setting the melt out of the melting furnace. The curves are in three sets. Each set consists of a solid, a dashed, and a dotted curve, showing the 
temperature regulation which resulted in good, fair, and poor annealing, respectively, for each of the three methods described below (Pittsburgh, Spencer, and Bausch \& Lomb). These curves were made on various types of glass and sizes of pots, and the character of the annealing was judged by three different men at three different plants. Thus the three sets cannot be directly compared.

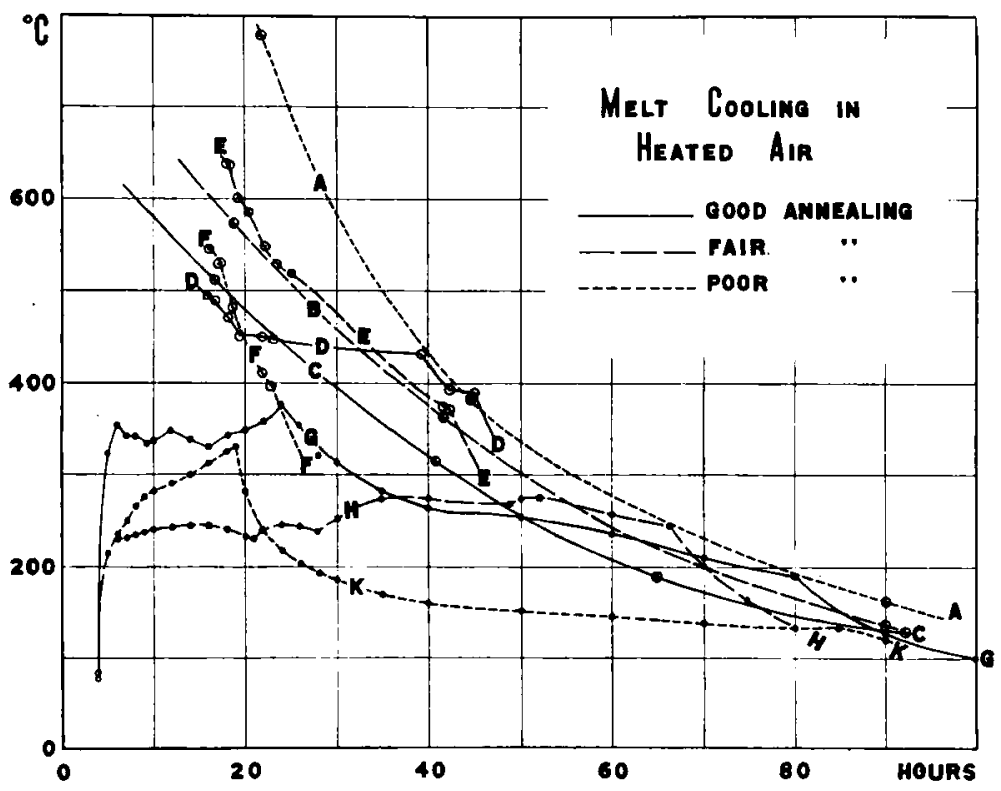

Frg. 5.-Time-temperature curves for melt cooling in heated air. The cooling was retarded by placing the pot in a heated pot-arch. The temperatures are those of the air over, and close to the pot.

Curves $\mathrm{A}, \mathrm{B}$, and $\mathrm{C}$ are plotted from data on the temperature of the air above the pot, taken at the Bausch \& Lomb plant, where the fire was kept burning in the arch until it had cooled to a low temperature. Each of these three curves was plotted from 3 (in one case 4) measurements, which accounts for their marked regularity. In spite of the fact that the lowest average cooling rate during the first three days was in the case of $\mathrm{A}$, nevertheless A gave the poorest annealing. Here the arch was 
kept for a long time at a high temperature, which greatly reduced the temperature gradient at this temperature, but did not prevent its reaching a high value as the melt passed rapidly through the annealing range. In the case of $B$ and $C$, the melt cooled rapidly at high temperatures, but the cooling was greatly retarded through the annealing range.

Curves D, E, and F represent data taken at the Spencer Lens plant where the gas was left burning until the arch had cooled to about $400^{\circ}$. This set also illustrates the fact that early retardation is not important in securing satisfactory annealing.

The data for Curves $\mathrm{G}, \mathrm{H}$, and $\mathrm{K}$ were taken at the Charleroi plant of the Pittsburgh Plate Glass Co.; they are part of the same series of experiments as Curves A, B, and C of Fig. 4. In the case of $\mathrm{G}$, while the annealing was entirely satisfactory, it was found later that the rate of cooling was slower than necessary. In the case of $\mathrm{H}$, a somewhat slower rate seemed desirable at the start. Where the curve was more nearly the mean of $\mathrm{G}$ and $\mathrm{H}$, it was found that a 36 -inch melt could be removed from the arch at the end of $2^{1 / 2}$ days, and broken at the end of 4 days. Curve $\mathrm{K}$ resulted, as was expected, in a marble. This schedule was designed to apply alike to all of the several types of glass made at that plant, and could therefore be somewhat shortened for individual cases.

Use of Insulation around the Melt.-Insulation may be applied to the pot either in the form of an insulating cover, or the pot may be surrounded by a layer of some loose insulating material such as sand or kieselguhr. Where sand is used, a thickness of 8 inches $(20 \mathrm{~cm}$.) is sufficient for pots 30 to 50 inches $(75$ to $I 20 \mathrm{~cm}$.) in diameter. This may be reduced, in the case of kieselguhr, to 3 inches $\left(7^{1 / 2} \mathrm{~cm}\right.$.).

The form of cover used at the Pittsburgh Plate Glass Company's plant consisted of a hollow double-walled sheet iron cylinder, closed at one end by a double wall. The 3 -inch $\left(7^{1 / 2} \mathrm{~cm}\right.$.) space between the walls was filled with kieselguhr, and two handles were riveted to the outside to facilitate handling. The height inside the cover was such that, when it was inverted over a pot, the bottom edge of the cover rested on the floor, and could be 
sealed with a little sand. The weight of such a cover for a 36 inch pot $(90 \mathrm{~cm}$.) was perhaps $150 \mathrm{lb} .(68 \mathrm{~kg}$.).

At the same plant, previous to the use of these covers, the melts were insulated with sand, held in place by a retaining case of sheet iron. These cases were made by riveting together two sheets of flat galvanized iron to form a continuous band. Their average diameter was greater than that of the pot, by 16 inches $(40 \mathrm{~cm}$.) or more, and their height was 30 inches $(75 \mathrm{~cm}$.$) . This$ height brought the top of the case to about the same level as the surface of the glass in the pot.

These cases were filled by shoveling the sand into them, an operation that consumed considerable time, as it involved moving about a ton of sand for each 36 -inch pot. When the sand was to be removed from the pot, the case was first lifted off by the crane, allowing the sand to form a cone around the bottom of the pot. This cone was then hoed back to clear the pot.

Of the two, the portable covers were preferable, entailing less time and labor and being more tidy. They were objected to at first on the ground that circulation in the air space between the pot and the cover might induce too rapid cooling. This was found not to be the case.

In Fig. 6 time-temperature curves are plotted for the cooling of two melts of medium flint glass $\left(n_{\mathrm{D}}=\mathrm{I} .6 \mathrm{I}\right.$ ) in 36 -inch (90 cm.) pots. In the case of Curves $A, B$, and $C$, the melt cooled freely for 5 hours after it was set out of the furnace; it was then insulated with a ro-inch $(25 \mathrm{~cm}$.) layer of sand retained by a sheet-iron case; this insulation was removed 62 hours after setting out. Curve $A$ represents the temperature at the center of the melt; Curve $B$, that at a point in contact with the inside of the pot midway between top and bottom; and Curve $C$, that on the outside of the pot opposite point B. The melt, sand and retaining case are drawn to scale in the upper part of the figure, and the location of the thermocouples shown by small circles. In the case of Curves $\mathrm{D}$ and $\mathrm{E}$, the melt was cooled in the open with no insulation other than a 4-inch ( 1 o $\mathrm{cm}$.) layer of kieselguhr on top. Curve $D$ is for a point in the melt corresponding to that of Curve A; Curve E, for a point corresponding to that of Curve E. These two curves are the Curves D and E of Fig. 4. 


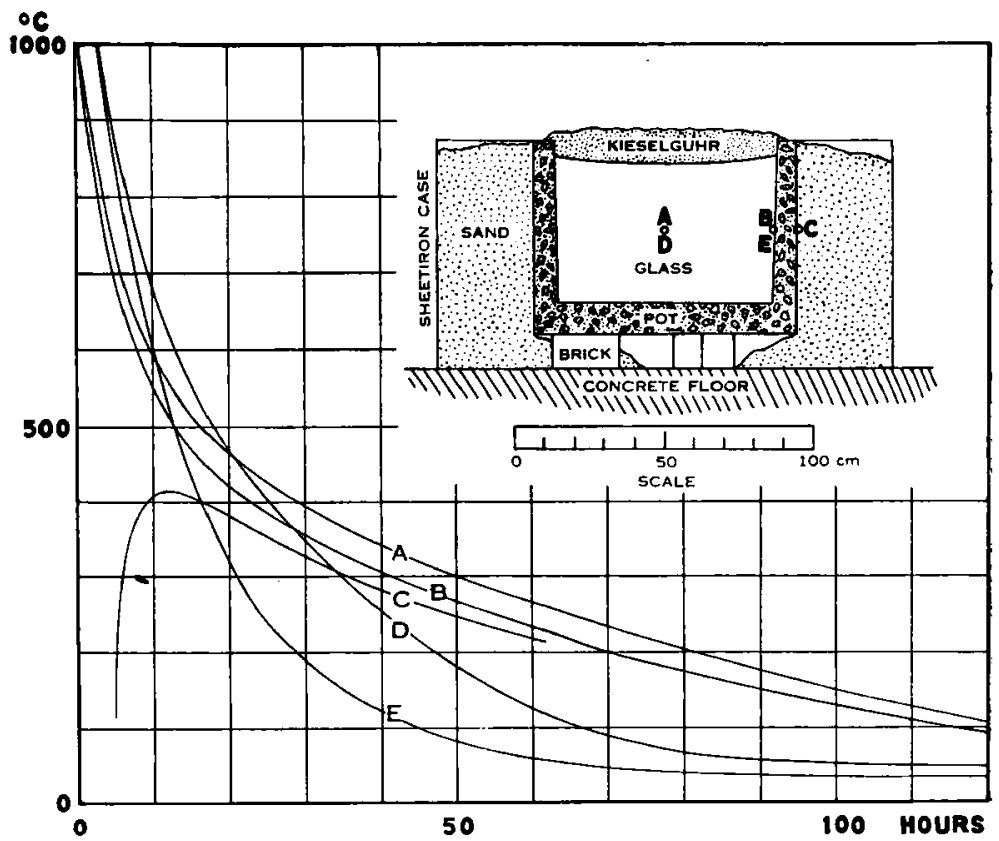

FIG. 6.-Time-temperature curves for a melt cooled in sand. Curves $D$ and $E$ of Fig. 4 are reproduced for comparison.

Course of the Temperature Drop.-In Fig. 7 the difference in temperature between the center and side of the melt, for the three

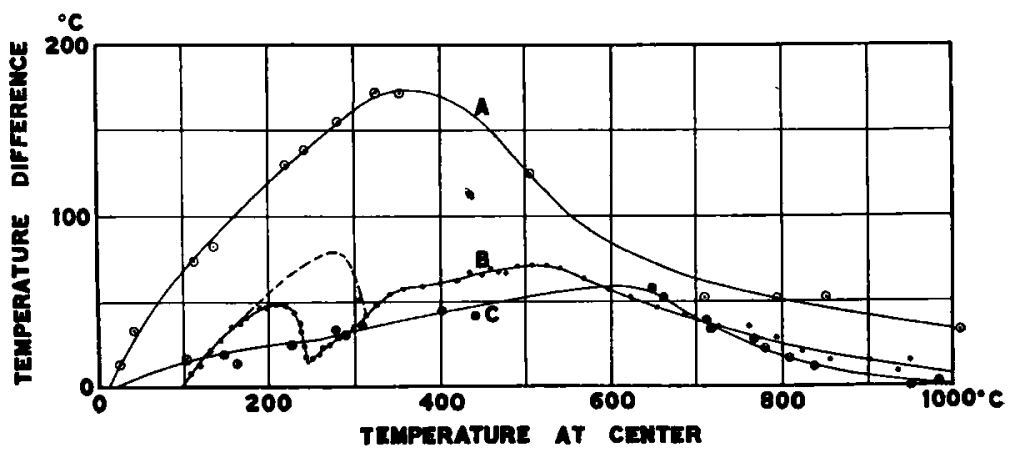

Fig. 7.-Temperature drop through the melt plotted against the corresponding temperature at the center, for the three melts of Fig. 4 and Fig. 6. 
melts of Fig. 4. and Fig. 6, are plotted against the corresponding temperatures at the center. Curve $A$ is for the melt cooled in the open (D and E of both Fig. 4 and Fig. 6); Curve B, for that cooled in a pot arch (A, B, and C of Fig. 4); Curve C, for that cooled in sand (A, B, and C of Fig. 6).

Most of the cracks in melt A were of the plane type, and there were many fragments weighing ro lbs. $\left(4^{1 / 2} \mathrm{~kg}\right.$.) or over. This may be explained by the fact that the temperature difference did not begin to increase until the outside of the melt had cooled to about $200^{\circ}$, a temperature well below that at which plane fracturing usually begins in this type of melt. However, the break could not be considered as satisfactory because of the large proportion of glass found broken into small fragments.

Curve $B$ shows two maxima. The first, at about 5 ro $^{\circ}$, was only attained after the center had cooled through more than $500^{\circ}$ at high temperatures; the second, at about $210^{\circ}$, was reached on shutting off the gas in the arch after the center had cooled through only $40^{\circ}$. This shows the very great role played by convection and radiation in reducing the temperature drop. The portion of the curve to the left of $25^{\circ}$ gives an idea of the extent to which the fall of the temperature drop can be postponed. In later experiments the gas was turned out in the arch much sooner, which would have caused the curve in this case to take a form something like that indicated by the dotted line in the figure. This practice resulted in as good a break and a very much shortened time of cooling.

The lower portion, below $260^{\circ}$, of Curve C shows a much smaller break than that of Curve $B$, probably because the cone of sand was not completely removed from that side of the pot-an experimental detail that was unfortunately not recorded.

Cooling Schedule, where Insulation is Applied to the Pot.The following schedule was developed by the author at the Charleroi plant of the Pittsburgh Plate Glass Company for cooling their 36-inch $(90 \mathrm{~cm}$.) pots and 49 -inch $(125 \mathrm{~cm}$.) pots. As each type of glass was set out of the melting furnace at its definite temperature, the operations of cooling were performed according to the time that had elapsed after the pot was set out. The 
schedule was so arranged that all pots of the same size were treated alike, regardless of the type of glass they contained.

Immediately on setting the melt out of the furnace, the surface of the glass was covered with a 4 -inch (ro $\mathrm{cm}$.) layer of kieselguhr and the pot placed on three bricks to cool.

Four hours later in the case of 36 -inch pots, and eight hours in the case of 49 -inch pots, insulation was applied to the pot in the form of an 8-inch $(20 \mathrm{~cm}$.) layer of loose sand or in the form of one of the covers described above.

The insulation was removed from the 36 -inch pots 3 days after setting out; and from the 49 -inch pots 5 days after setting out. Two or three days later the melts were cool enough to be broken down.

Where 36 -inch melts are cooled in sand according to this schedule the very approximate temperatures given in Table I may be expected at the center of the melt. The third and fifth columns of numbers refer to the times insulation is applied and removed, respectively. Some of the annealing temperatures given in the fourth column were determined by G. W. Morey, of the Geophysical Laboratory, using the rough method given above; the others were extrapolated from data obtained for the release of strain at lower temperatures by L. H. Adams and E. D. Williamson, of this laboratory.

TABLE I.

Approximate Temperatures at Center of 36 -inch Melts Cooled in Sand.

\begin{tabular}{|c|c|c|c|c|c|}
\hline Type of glass. & $n_{\mathrm{D}}$ & $\begin{array}{l}\text { Temp. } \\
\text { set out. }\end{array}$ & $\begin{array}{c}\text { Temp. } \\
\text { after } \\
4 \text { hrs. }\end{array}$ & $\begin{array}{c}\text { Anneal- } \\
\text { ing } \\
\text { temp. }\end{array}$ & $\begin{array}{l}\text { Temp. } \\
\text { after } \\
72 \text { hrs. }\end{array}$ \\
\hline Flint. & I. 57 & $1038^{\circ}$ & $800^{\circ}$ & $465^{\circ}$ & $320^{\circ}$ \\
\hline Flint. . . . & 1.61 & 996 & 780 & 455 & 300 \\
\hline Flint....... & I. 66 & 968 & 760 & 445 & 290 \\
\hline Crown, Ordinary ..... & I. 52 & 1093 & 850 & 570 & 350 \\
\hline Crown, Borosilicate... . & I. 52 & I I I 6 & 860 & 590 & 360 \\
\hline Crown, Barium....... & I. 57 & 1016 & 790 & 580 & 3 Io \\
\hline Crown, Barium........ & I. $6 I$ & 1016 & 790 & 600 & 310 \\
\hline
\end{tabular}

Breaking Down.-When the melt is ready to be broken down, the diametral crack in the glass is first located, and the pot broken 
at the ends of it with a sledge. The two halves of the melt are now separated and the blocks of glass loosened by beating on the outside of the pot, or shaking it with a crow bar. A small hammer is used where necessary to free the blocks of adhering pot shell.

\section{Summary.}

The conditions to be attained when a melt of optical glass is cooled in the pot are: (I) that neither ream nor bubbles shall be introduced during the cooling, nor carried into the middle of the melt; (2) that the glass shall not become inhomogeneous through the precipitation of a crystalline phase; (3) that the bulk of the cooled melt shall be found cracked into large, reasonably rectangular blocks, having smooth, flat surfaces; and (4) that these blocks shall be sufficiently free from strain to cleave readily with a smooth fracture.

The appearance of ream in the middle of the melt, vacuum bubbles and a crystalline phase, can be discouraged by rapid cooling, preferably from the bottom of the pot, while the glass is still soft; and by insulating its top surface as soon as the melt is set out of the melting furnace.

The cold melt shows cracks of two types: "spherical cracks," and "plane cracks." The spherical cracks are due to decrease of temperature difference, and first appear below the temperature at which the drop is a maximum; these cracks form one after another until the temperature gradient has disappeared. The plane cracks seem to be the result of a difference in rate of contraction between the glass and the pot; the temperature at which they first appear in a given melt seems to be nearly independent of the cooling rate. The presence of either type of crack in the melt reduces the tendency of the other type of crack to form. As the fracture due to spherical cracks is rough and the pieces formed are irregular, while that due to plane cracks is entirely satisfactory, it is desirable ( $\mathrm{I}$ ) to maintain the temperature gradient at a low value by slow cooling; and (2) to prevent its decreasing much below its maximum until after the formation of plane cracks has begun, by increasing the cooling rate at the proper time. 
The cooling rate can be reduced ( $I$ ) by heating the surroundings of the melt, i.e., placing it in a heated kiln; and (2) by surrounding it with an envelop of some such insulating material as sand, kieselguhr, etc. Variations of these two methods are described and time-temperature data given.

GFOPHYSICAI, LABORATORY,

Carnegre Institution of Washington, April, 1919. 